\title{
EFFECT OF NEUROMUSCULAR RESISTANCE TRAINING ON THE PERFORMANCE OF 5-KM RUNNERS
}

\author{
Miller P. Guimarães ${ }^{1,2,3}$, Yuri A.C. Campos ${ }^{3,4}$, Hiago L.R. de Souza ${ }^{3,4}$, \\ Gaspar P. da Silva ${ }^{3}$, Claudio Hernández-Mosqueira ${ }^{5,6}$, and Sandro F. da Silva ${ }^{3}$ \\ ${ }^{1}$ Federal University of São Paulo, Santos, Brazil \\ ${ }^{2}$ Presbyterian College Gammon, Lavras, Brazil \\ ${ }^{3}$ Federal University of Lavras, Lavras, Brazil \\ ${ }^{4}$ Faculty of Physical Education and Sports of the University of Juiz de fora, \\ Federal University of Juiz de Fora, Juiz de Fora, Brazil \\ ${ }^{5}$ Department of Physical Activity Sciences, University of Los Lagos, Puerto Montt, Chile \\ ${ }^{6}$ AFSYE research group, Adventist University of Chile, Chile
}

Original scientific article

DOI: $10.26582 / \mathrm{k} .52 .1 .8$

\begin{abstract}
:
The study aimed to identify the effect of a neuromuscular resistance training protocol (NRTP) on the performance of 5-km distance runners. This study included 18 male runners (age $=29.3 \pm 3.2$ years, fat percentage $=11.3 \pm 2.6 \%$, body height $=1.77 \pm .04 \mathrm{~m}$, body mass $=73.4 \pm 4.4 \mathrm{~kg}$, time in $5 \mathrm{~km}=20.6 \pm 2.4 \mathrm{~min}$, training experience $=4.3 \pm 0.7$ years). First, volunteers were anthropometrically evaluated, and they performed one-repetition maximum (1RM) $45^{\circ}$ leg press (LP) strength test. Second, they performed an incremental protocol in the $45^{\circ} \mathrm{LP}$ to acquire the electromyographic threshold. Third, they completed a $5-\mathrm{km}$ time trial run $(5 \mathrm{~km}$ basal). In the fourth session, they performed NRTP in LP. And fifth, the 5-km time trial run was performed at $30 \mathrm{~min}, 48 \mathrm{~h}, 96 \mathrm{~h}$, and $144 \mathrm{~h}$ post the NRTP intervention. A significant decrease $(\mathrm{p} \leq .05)$ was observed when baseline values were compared with post $30 \mathrm{~min}$ and post $48 \mathrm{~h}$ ( $p=.02$ and $p=.04$, respectively). However, there were significant positive differences in performance ( $\mathrm{p}=.04$ for time) when baseline values and post $144 \mathrm{~h}$ were analyzed. Therefore, it is concluded that the NRTP can be used by $5-\mathrm{km}$ distance runners to improve their performance with a break of one week between the intervention and test.
\end{abstract}

Key words: performance, endurance, physiology and exercise

\section{Introduction}

The performance of runners can be described as a multifactorial phenomenon (Beattie, Carson, Lyons, Rossiter, \& Kenny, 2017), although several researchers have leveraged the importance of physiological and biomechanical factors to the performance of this population of endurance athletes (Balsalobre-Fernández, Santos-Concejero, \& Grivas, 2016; Beattie, et al., 2017; Denadai, de Aguiar, de Lima, Greco, \& Caputo, 2016; Enoksen, Shalfawi, \& Tønnessen, 2011; Lum, Tan, Pang, \& Barbosa, 2016; Mikkola, et al., 2011; Schumann, Pelttari, Doma, Karavirta, \& Häkkinen, 2016; Taipale, et al., 2010). Therefore, the understanding of the manipulation and control of the physiological indexes associated to the performance of endurance runners is of utmost importance in making them useful tools for the training prescription (Camic,
Hahn, Hendrickson, \& Jagim, 2017; Guglielmo, Babel Junior, Arins, \& Dittrich, 2012; ), specifically in middle-distance runners, in whom performance is determined primarily by the manipulation of aerobic and anaerobic capacities (Enoksen, et al., 2011) and its prevalence at each stage of a test (Denadai, Ortiz, \& Mello, 2004).

Several training modality proposals have been found in literature (Enoksen, et al., 2011; Lum, et al., 2016; Mikkola, et al., 2011). Basically, the prescription of endurance running training methods usually embraces specific training (Enoksen, et al., 2011), or complementary training that uses maximum strength, explosive strength, and plyometric exercises (Beattie, et al., 2017; Paavolainen, Häkkinen, Hämäläinen, Nummela, \& Rusko, 1999). Thus, the combination of these two types of training modalities seems to be a positive strategy to improve the 
running economy (RE) of these athletes (Barnes \& Kilding, 2015; Mikkola, et al., 2011; Taipale, et al., 2010) and hence their performance, since athletes with a better running economy have lower blood lactate concentration and oxygen uptake at submaximal intensities when compared to the athletes who have even higher $\mathrm{VO}_{2} \max$ (Billat, et al., 2003).

Thus, recent studies have also emphasized the applicability of resistance training (RT) to improve running performance and obtained significant results concerning RE in recreational (Taipale, et al., 2010; Taipale, Mikkola, Vesterinen, Nummela, \& Häkkinen, 2013), moderately-trained (Guglielmo, Greco, \& Denadai, 2009), and highly-trained athletes (Balsalobre-Fernández, et al., 2016; Sedano, Marín, Cuadrado, \& Redondo, 2013). The obtained increases in running performance may be related to numerous factors, such as improved coordination and co-activation among the lower limb muscles that hence increase leg stiffness, providing a shorter transition time between the stages of braking and propulsion during running (Cheng, et al., 2012); or positive adaptive responses in the recruitment and synchronization of motor units (MUs), reflecting the efficiency and mechanics of motor recruitment patterns (Rønnestad \& Mujika, 2014) and positive changes in the biomechanical aspects of running, which would result in a representative decrease in oxygen consumption at submaximal intensities and muscle fatigue retardation (Hanon, ThépautMathieu, \& Vandewalle, 2005).

Other studies that evaluated running performance over distances of $5 \mathrm{~km}$ (Hudgins, Scharfenberg, Triplett, \& McBride, 2013; Paavolainen, et al., 1999) and $10 \mathrm{~km}$ (Damasceno, et al., 2015) and its relationships with RT, we noticed variations mainly in RT volumes and intensities, which represent different manifestations of strength (explosivestrength and endurance-strength). In this sense, prescription of RT exercises with loads referring to the electromyographic threshold (EMGT), may reflect stimuli different from those commonly reported (for example, Hug, Laplaud, Lucia, \& Grelot, 2006). Thus, even if the adaptive principles are the same as in the studies cited here (Arampatzis, et al., 2006; Cheng, et al., 2012; Markovic \& Mikulic, 2010; Nummela, et al., 2006; Saunders,
Pyne, Telford, \& Hawley, 2004), it is not yet clear in literature what is the influence of EMGT intensity on the performance of long-distance runners.

Acute adaptations coming specifically from RT, such as mechanisms of muscle fiber damages (Chen, Lin, Chen, Lin, \& Nosaka, 2011) and neural factors (Aagaard, et al., 2000) can trigger either positive or negative responses in the athlete's performance, influenced by both recovery time and training volume. This relationship between stimulus and recovery becomes a primary alternative in the search for performance improvement and in the complementation of specific training of endurance running (Garnacho-Castaño, Domínguez, Ruiz-Solano, \& Maté-Muñoz, 2015). However, in current literature, there are no studies with RT that developed works with a high volume of sessions and intensity at the EMGT, using the $45^{\circ}$ leg press (LP) as the training contents. Therefore, the present study aimed to identify the effect of an acute neuromuscular resistance training protocol (NRTP) on the performance of 5-km distance runners. The hypothesis was that the NRTP would have positive effects on the performance of 5-km distance runners.

\section{Methods}

\section{Participants}

Eighteen male experienced runners, moderately trained (age $=29.27 \pm 3.24$ years, fat $\%=11.33 \pm 2.62$, body height $=1.77 \pm 0.04 \mathrm{~m}$, body mass $=73.44 \pm 4.38$ $\mathrm{kg}$, time in $5 \mathrm{~km}=20.63 \pm 2.35 \mathrm{~min}$, running training experience $=4.26 \pm 0.68$ years), with experience in resistance training (minimum two years) and without any osteoarticular lesion participated in the study. Table 1 shows the weekly training model of the runners. All volunteers were pre-informed about all risks involved in their participation in the research and had previously signed the free and clarified consent form. During the study, participants were instructed to refrain from other exercises or resistance training. The study protocol was approved by the Ethics Committee (CAAE Number: 53675416.3.0000.5148) following directives of the last revision of the Declaration of Helsinki.

Table 1. Weekly training model of the runners

\begin{tabular}{|c|c|c|c|c|c|c|}
\hline \multicolumn{7}{|c|}{ VAT: $14.5 \mathrm{~km} / \mathrm{hr} \mathrm{vVO}_{2 \max }: 16.3 \mathrm{~km} / \mathrm{hr}$} \\
\hline Monday & Tuesday & Wednesday & Thursday & Friday & Saturday & Sunday \\
\hline $\begin{array}{l}5 \text {-min specific running } \\
\text { warm-up at } 70 \% \text { of } \\
\text { VAT }+15 \times 500 \mathrm{~m} \text { in } \\
\text { the } \mathrm{VVO}_{2 \max } \text { by }(60: 90 \\
\text { seconds of passive } \\
\text { recovery) }\end{array}$ & $\begin{array}{l}\text { Gym center: Leg } \\
\text { Press, Squat, } \\
\text { Stiff and Plantar } \\
\text { Flexion: } 4 \mathrm{x} \text { in the } \\
\text { exhaustion mode } \\
70 \% \text { of } 1 \mathrm{RM}\end{array}$ & $\begin{array}{l}5 \text {-min specific } \\
\text { running warm-up } \\
\text { at } 70 \% \text { of VAT + } \\
90 \text { minutes race in } \\
\text { the VAT on a flat } \\
\text { terrain }\end{array}$ & $\begin{array}{l}\text { Gym center: Leg } \\
\text { Press, Squat, } \\
\text { Stiff and Plantar } \\
\text { Flexion: } 4 \text { x in the } \\
\text { exhaustion mode } \\
70 \% \text { of } 1 \mathrm{RM}\end{array}$ & Rest & $\begin{array}{l}5-\mathrm{km} \text { race } \\
\text { in the time- } \\
\text { trial on } \\
\text { track }\end{array}$ & Rest \\
\hline
\end{tabular}

Note. VAT - velocity at the anaerobic threshold; $\mathrm{VVO}_{2} \mathrm{max}$ - velocity in the $\mathrm{VO}_{2} \mathrm{max}$; $\mathrm{RM}$ - repetition maximum. 


\section{Experimental design}

Participants performed sessions in the laboratory and on a running track on five distinct occasions that were divided into control variables (sessions 1 and 2) and performance variables (sessions 3, 4, and 5). In the first session, anthropometric assessment and the one-repetition maximum (1RM) test were performed in the laboratory to determine the maximum load in the LP exercise. In the second session ( $48 \mathrm{~h}$ after the 1st one) the incremental load protocol in the LP was implemented for the EMGT determination. In the third session $(72 \mathrm{~h}$ after the 2nd one), the $5-\mathrm{km}$ time trial run was performed. This so-called "pre-intervention" test served to normalize track-related data and for future comparisons. In the fourth session ( $48 \mathrm{~h}$ after the $3 \mathrm{rd}$ one) the NRTP in the LP device was performed with individually tailored loads, predetermined in session 2. Finally, the fifth session, which was divided into post $30 \mathrm{~min}$, post $48 \mathrm{~h}$, post $96 \mathrm{~h}$ and post $144 \mathrm{~h}$ after the fourth session, i.e. intervention, consisted of the four $5-\mathrm{km}$ time trial runs, which trials repeated the same procedure of the third session. All tests (laboratory and track alike) were executed in similar climatic conditions (temperature $=23-27^{\circ} \mathrm{C}$, relative humidity $=60-68 \%$ ). Verbal encouragements were given during all tests. The experiment was concluded in two weeks, with all the tests executed at the same time of day. The description of methods is exemplified in Figure 1.

\section{Anthropometric characteristics and body composition}

Body mass and height of the participants were measured using a scale with stadiometer Welmy ${ }^{\circledR}$. Body composition was determined by the bioimpedance test using a tetrapolar device (Quantum BIA-II, RJL Systems ${ }^{\circledR}$, Clinton Township, USA) with the Body Composition 2.1 software.

\section{RM test}

The identification of the maximum dynamic force was done through the 1RM test in the $45^{\circ}$ LP exercise (Mark BS Fitness $\left.{ }^{\circledR}\right)$. Individuals were initially positioned with their feet and extended knees aligned at the hip width. The execution started with the eccentric phase of the movement and was developed up to a $90^{\circ}$ angle at the knee. From this position, the concentric phase of the movement began until the knees were fully extended. Before the test, a warm-up (six to 10 repetitions) was performed with approximately $50 \%$ of the estimated load for the first attempt on the 1RM test. After two minutes of rest, the test started. Participants were instructed to complete two repetitions. If the two repetitions were completed on the first attempt or even if a participant did not complete a single repetition, the second attempt was made after a recovery interval of 3-5 min with a higher (first possibility) or lower load (second possibility) to that employed in the previous attempt. This procedure was repeated on the third and fourth attempt. The load recorded as $1 \mathrm{RM}$ was one in which each individual was able to complete a single maximal repetition (Brown \& Weir, 2001). During the test, the movement velocity was controlled with a digital metronome (DM90, Seiko ${ }^{\circledR}$, Tokyo, Japan), being $2 \mathrm{~s}$ for the eccentric phase and $1 \mathrm{~s}$ for the concentric phase.

\section{Incremental protocol performed on $45^{\circ}$ LP}

After obtaining individual load values in the $1 \mathrm{RM}$ test, they were fractionated in $10,20,25,30$, $35,40,50,60,70,80$, and $90 \%$ of $1 \mathrm{RM}$. In order to perform the incremental load protocol, volunteers executed progressive load stages starting with an initial load of $10 \% 1 \mathrm{RM}$ and terminated their performance at the moment when they failed to perform the movement within the correct estab-

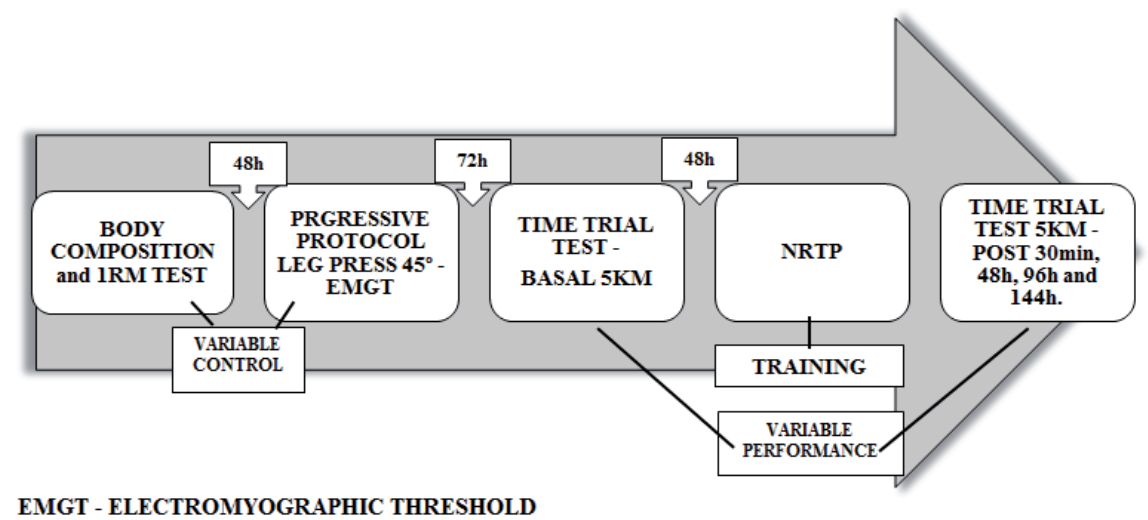

EMGT - ELECTROMYOGRAPHIC THRESHOLD

NRTP - NEUROMUSCULAR RESISTANCE TRAINING PROTOCOL

Figure 1. Study design. 
lished biomechanical pattern, or due to the inability to perform the determined number of repetitions. The total duration of each stage was one minute or 20 repetitions, with the movement speed controlled by a digital metronome (DM90, Seiko ${ }^{\circledR}$, Tokyo, Japan), being adopted $1 \mathrm{~s}$ for the concentric phase and $2 \mathrm{~s}$ for the eccentric phase. An interval of $2 \mathrm{~min}$ was observed between each stage for recovery and readjustment of workloads for the next stage (de Sousa, et al., 2011). The purpose of the incremental load protocol was to obtain the individual load value corresponding to the electromyographic threshold that would subsequently be used in the NRTP also in the LP device.

\section{Electromyography and electromyographic threshold}

As the main objective of the incremental load protocol was the acquisition of the load corresponding to the EMGT, electromyographic collections were made in the following manner. The following muscles were evaluated: $m$. rectus femoris (RF), $m$. vastus lateralis (VL), and $m$. vastus medialis (VM). To avoid possible interference in the electromyographic signal, participants' skin was prepared through the processes of excess hair removal, abrasion, and cleaning with cotton and isopropyl alcohol. Electromyographic electrodes were $2223 \mathrm{BR}, 3 \mathrm{M} \AA$, Campinas, Brazil, with coupled conducting gel, $1 \mathrm{~cm}$ diameter $\mathrm{AgCl}$ capture surface, flat round disc. They were placed in the presumable direction of the underlying muscle fibers with a center-to-center distance of approximately $2 \mathrm{~cm}$. The surface electrodes were positioned on the respective muscles according to SENIAM recommendations (Hermens, et al.,1999) and fixed on the dominant side of the volunteers (Behm, Leonard, Young, Bonsey, \& MacKinnon, 2005). The reference electrodes were properly placed in the bony process of the patella. An electromyograph (Miotool 400, Miotec ${ }^{\circledR}$ Biomedical Equipments Ltda., Porto Alegre, Brazil), with four input channels, 14 bits of resolution and an acquisition rate per channel of 2000 samples/s, with an SDS-500 sensor with a maximum gain of 1000 times, was used to collect the electromyographic signal. The common mode of rejection rate was $106 \mathrm{~dB}$ and the impedance between each electrode pair was $<10^{12} \Omega$. All electromyograph channels were properly calibrated before data collection. After the collection, the electromyographic signals were filtered through the fifth-order Butterworth low-pass filter with a cut-off frequency of 20-500 Hz. The amplitude of signals was expressed with the root mean squares (RMS). The data were normalized based on the peak channel means. To determine the EMGT in the RMS, the mathematical model was adopted using the RMS data in a simple regression equation, which divides the data into two straight lines and the point of the greatest distance was determined as the EMGT (Hug, et al., 2006).

\section{Neuromuscular resistance training protocol (NRTP) - Intervention}

Acute neuromuscular resistance training (adapted from Garnacho-Castaño, et al., 2015) in the LP was performed $48 \mathrm{~h}$ after the performance evaluation of 5-km running (pre-intervention) and its intensity corresponded to the electromyographic threshold obtained in the incremental load test. The test started with a warm-up pattern consisting of 5 min of light jogging and 5 min of joint mobility exercises and ballistic stretching of the lower limbs. The constant loading protocol consisted of 21 sets of 15 repetitions. The execution speed was $3 \mathrm{~s}(1 \mathrm{~s}$ for concentric and $2 \mathrm{~s}$ for eccentric phase), with the movement speed controlled with a digital metronome. The 1-min recovery among the series was passive (Garnacho-Castaño, et al., 2015).

\section{Five-km time trial-Pre- and post- intervention}

These evaluations consisted of running $5 \mathrm{~km}$ in the shortest possible time. The pre-intervention test (session 3) was performed $48 \mathrm{~h}$ before the NRTP. The post-intervention tests were performed $30 \mathrm{~min}$, $48 \mathrm{~h}, 96 \mathrm{~h}$, and $144 \mathrm{~h}$ after the NRTP implementation. The final times of each participant were collected for all 5-km performance evaluations and the volunteers did not receive any feedback about the test time or the traveled distance. The test was performed on a $400-\mathrm{m}$ track containing nine lanes measuring approximately $1.25 \mathrm{~m}$ in width. During the test, the researcher was responsible for starting the test, noting the number of laps, timing, and closing the test. For the timing, a digital stopwatch was used (S141, Seiko ${ }^{\circledR}$, Tokyo, Japan).

\section{Statistical analysis}

A descriptive analysis was used with the study of means and standard deviation. Levene's test was used to verify the sampling distribution and parametric tests were used due to the sampling homogeneity. For the time variable analysis before and after the resistance protocol, the ANOVA for repeated measurements test was used and the Tukey's posthoc test. For the analysis of effect size, Cohen's F test was used. A $\mathrm{p} \leq .05$ was used for statistical proof. All the statistical analyses were performed by the SPSS 21.0 software.

\section{Results}

The study results are described below. Table 2 shows the mean times in minutes for the different test sessions performed on the track and the values of the statistical differences of comparisons made among the $5-\mathrm{km}$ mean times in the different sessions. 
Table 2. Mean time and time comparison of all times of run tests with $p$ values

\begin{tabular}{|c|c|c|c|c|c|}
\hline & $\mathrm{T}_{\mathrm{PRE}}$ & $\mathrm{T}_{30 \min }$ & $\mathrm{T}_{48 \mathrm{~h}}$ & $\mathrm{~T}_{96 \mathrm{~h}}$ & $T_{144 \mathrm{~h}}$ \\
\hline Time (min): $\mathrm{M} \pm \mathrm{SD}$ & $20.63 \pm 2.42$ & $22.40 \pm 2.86$ & $21.26 \pm 2.56$ & $20.58 \pm 2.40$ & $20.23 \pm 2.33$ \\
\hline $\mathrm{T}_{\mathrm{PRE}}$ & & $.02^{*}$ & $.04^{*}$ & .07 & $.04^{*}$ \\
\hline $\mathrm{T}_{30 \min }$ & & & $.02^{*}$ & $.03^{*}$ & $.03^{*}$ \\
\hline $\mathrm{T}_{48 \mathrm{~h}}$ & & & & $.04^{*}$ & $.03^{*}$ \\
\hline $\mathrm{T}_{96 \mathrm{~h}}$ & & & & & $.04^{*}$ \\
\hline $\mathrm{T}_{144 \mathrm{~h}}$ & & & & & \\
\hline
\end{tabular}

"Indicates a significant difference between moments.

The average time values for the entire sample were: $20.63 \pm 2.42 \mathrm{~min}$ for the pre-intervention or basal test; $22.40 \pm 2.86 \mathrm{~min}$ in post $30 \mathrm{~min} ; 21.26 \pm 2.56$ min in post $48 \mathrm{~h} ; 20.58 \pm 2.40 \mathrm{~min}$ in post $96 \mathrm{~h}$; and $20.23 \pm 2.33 \mathrm{~min}$ in post $144 \mathrm{~h}$. Regarding the effect size analysis, which was generally large, we can conclude that the sample size did not show any interference in the results (Tbasal $=.89, \mathrm{~T} 30=.88$, $\mathrm{T} 48=.79, \mathrm{~T} 90=.84$, and $\mathrm{T} 144=.89$ ).

\section{Discussion and conclusions}

The main finding of our study was that the NRTP improved the 5-km performance of moderately trained runners based on the comparison of times between baseline and $144 \mathrm{~h}$ post-intervention. It was also verified that a significant decrease in performance was observed when comparing the baseline time with the $30 \mathrm{~min}$ and $48 \mathrm{~h}$ postintervention sessions, which was not observed between the baseline and post $96 \mathrm{~h}$ session since no statistical difference was found in this comparison. The observed changes in performance after NRTP can be explained by neural (Aagaard, et al., 2000; Häkkinen, et al., 2003; Nummela, et al., 2006), mechanical (Oliveira, et al., 2013), and metabolic factors (Garnacho-Castaño, et al., 2015). Another important factor for the understanding of both performance improvement and worsening is to comprehend acute muscle fatigue and its impact on physiological adaptations (Hanon, et al., 2005).

Elucidating neural patterns, the performance decrease in $30 \mathrm{~min}$ and $48 \mathrm{~h}$ after the NRTP may be related to the study by Girard, Millet, Micallef, and Racinais (2012). These authors assessed the neuromuscular properties of runners after a $5-\mathrm{km}$ time trial test and found that the athletes had a $27 \%$ decrease in maximal isometric voluntary contraction after the intervention compared to the previous contraction. Thus, a direct relationship of the cited study with the present study is created, where the reduction of strength/performance can be explained by peripheral adjustments attributed to neuromuscular transmission failure and excitation-contraction coupling. This fatigue may also have decreased the plexus of efferent pathways between the vertebral column and muscles of the lower limbs, which inhibits the excitability of motor neurons (Girard, et al., 2012). Regarding the mechanical and metabolic factors responsible for the negative adaptations of performance, we identified that the NRTP might have caused mechanical and metabolic stress in individuals, which would lead to a decrease in their performance. An explanation for the stress is the theory of muscle damage. Such a mechanism is expressed by the rupture of the $\mathrm{Z}$ line (Clarkson \& Hubal, 2002) by sarcomere misalignment (McHugh, 2003) and by the damage of the sarcoplasmic reticulum. The study by Garnacho-Castaño et al. (2015) clarifies this point since authors found a significant decrease in strength and power parameters induced by muscle fatigue through using 21 sets of 15 repetitions in the squat exercise.

However, when the RT stimulus/recovery ratio (such as maximum strength, explosive strength, plyometric training, and combined training) respects the principle of general adaptation syndrome, it can bring significant improvements in runner's performance (Balsalobre-Fernández, et al., 2016; Beattie, et al., 2017; Denadai, et al., 2016; Lum, et al., 2016; Mikkola, et al., 2011; Schumann, et al., 2016; Taipale, et al., 2010; Vorup, et al., 2016). In line with the above-mentioned authors' inferences that the adaptation (gain in strength) originates primarily from neural adaptations, we can attribute the significant performance improvement in the post $144 \mathrm{~h}$ session, when compared to baseline, to the improvement in neural control and improvement in coordination and co-activation of the lower limbs' musculature (Cheng, et al., 2012; Nummela, et al., 2006), to the improvement of central and peripheral control through an advance of pre-activation (muscle activation before foot impact with the soil and reflex facilitation during the late eccentric and early concentric phases), as well as to the improvement in the stretch reflex, which resulted in the improvement of the reactive force (Markovic \& Mikulic, 2010). Other factors related to positive changes from baseline to post $144 \mathrm{~h}$ can also be explained by the improvement of the stretch/shortening cycle, which may improve the elastic energy optimization (Saunders, et al., 2004) and hence improve the running economy of 
athletes (Arampatzis, et al., 2006), i.e. this type of training would increase the importance of elastic components through stiffness of the muscle-tendon complex, since studies suggest that a stiff muscle allows a greater and faster use and transmission of strength (faster storage and release of energy) (Markovic \& Mikulic, 2010).

In addition to the previous explanations for the NRTP adaptations, the level of physical conditioning and the total training time of the evaluated group (Chen, et al., 2011; McHugh, 2003) would have a direct relationship with the theory of the protective effect of repeated loading, defined as lower fatigue or muscle damage overtime after the first stimulus (Paschalis, et al., 2008). Based on this, it is believed that the sample would suffer just minor interferences of the own lane tests, oppo- site to the idea that the own 5-km track evaluations could interfere with the results.

A possible limitation of the study was the lack of a control group. Nevertheless, the study by Bosak et al. (2009) revealed that a break of more than $24 \mathrm{~h}$ and less than $72 \mathrm{~h}$ would be sufficient for runners to recover from a simulated 5-km running test (Bosak, Bishop, Green, Hawver, \& Iosia, 2009). Therefore, the recovery period adopted in the present study (48 h) fits in this way, reinforcing the counterposition that the own lane $5-\mathrm{km}$ track tests could interfere with the results.

In conclusion, the NRTP can be used by moderately trained and experienced 5-km runners in track practices to improve their performances with a 144-h break between the stimulus and the test.

\section{References}

Aagaard, P., Simonsen, E., Andersen, J., Magnusson, S., Halkjaer-Kristensen, J., \& Dyhre-Poulsen, P. (2000). Neural inhibition during maximal eccentric and concentric quadriceps contraction: Effects of resistance training. Journal of Applied Physiology, 89(6), 2249-2257.

Arampatzis, A., De Monte, G., Karamanidis, K., Morey-Klapsing, G., Stafilidis, S., \& Brüggemann, G.-P. (2006). Influence of the muscle-tendon unit's mechanical and morphological properties on running economy. Journal of Experimental Biology, 209(17), 3345-3357.

Balsalobre-Fernández, C., Santos-Concejero, J., \& Grivas, G.V. (2016). Effects of strength training on running economy in highly trained runners: A systematic review with meta-analysis of controlled trials. Journal of Strength and Conditioning Research, 30(8), 2361-2368.

Barnes, K.R., \& Kilding, A.E. (2015). Strategies to improve running economy. Sports Medicine, 45(1), 37-56.

Beattie, K., Carson, B.P., Lyons, M., Rossiter, A., \& Kenny, I.C. (2017). The effect of strength training on performance indicators in distance runners. Journal of Strength and Conditioning Research, 31(1), 9-23.

Behm, D.G., Leonard, A.M., Young, W.B., Bonsey, W.A.C., \& MacKinnon, S.N. (2005). Trunk muscle electromyographic activity with unstable and unilateral exercises. Journal of Strength and Conditioning Research, 19(1), $193-201$.

Billat, V., Lepretre, P.-M., Heugas, A.-M., Laurence, M.-H., Salim, D., \& Koralsztein, J.P. (2003). Training and bioenergetic characteristics in elite male and female Kenyan runners. Medicine and Science in Sports and Exercise, 35(2), 297-304.

Bosak, A., Bishop, P., Green, J.M., Hawver, G., \& Iosia, M. (2009). Comparison of 5km running performance after 24 and 72 hours of passive recovery. Sport Journal, 12(4).

Brown, L.E., \& Weir, J.P. (2001). ASEP procedures recommendation I: Accurate assessment of muscular strength and power. Professionalization of Exercise Physiology, 4(11).

Camic, C.L., Hahn, C.J., Hendrickson, B.C, \& Jagim, A.R. (2017). Validation of the physical working capacity at the fatigue threshold treadmill test. Kinesiology, 49(2), 146-152.

Chen, T.C., Lin, K.-Y., Chen, H.-L., Lin, M.-J., \& Nosaka, K. (2011). Comparison in eccentric exercise-induced muscle damage among four limb muscles. European Journal of Applied Physiology, 111(2), 211-223.

Cheng, C.-F., Cheng, K.-H., Lee, Y.-M., Huang, H.-W., Kuo, Y.-H., \& Lee, H.-J. (2012). Improvement in running economy after 8 weeks of whole-body vibration training. Journal of Strength and Conditioning Research, 26(12), 3349-3357.

Clarkson, P.M., \& Hubal, M.J. (2002). Exercise-induced muscle damage in humans. American Journal of Physical Medicine and Rehabilitation, 81(11), S52-S69.

Damasceno, M.V., Lima-Silva, A.E., Pasqua, L.A., Tricoli, V., Duarte, M., Bishop, D.J., \& Bertuzzi, R. (2015). Effects of resistance training on neuromuscular characteristics and pacing during $10-\mathrm{km}$ running time trial. European Journal of Applied Physiology, 115(7), 1513-1522.

Denadai, B.S., de Aguiar, R.A., de Lima, L.C.R., Greco, C.C., \& Caputo, F. (2016). Explosive training and heavy weight training are effective for improving running economy in endurance athletes: A systematic review and meta-analysis. Sports Medicine, 47, 545-554. 
Denadai, B.S., Ortiz, M.J., \& Mello, M.D. (2004). Physiological indexes associated with aerobic performance in endurance runners: Effects of race duration. Revista Brasileira de Medicina do Esporte, 10(5), 401-404.

de Sousa, N.M.F., Magosso, R.F., Pereira, G.B., Leite, R.D., Arakelian, V.M., Montagnolli, A.N., . ., \& Baldissera, V. (2011). The measurement of lactate threshold in resistance exercise: A comparison of methods. Clinical Physiology and Functional Imaging, 31(5), 376-381.

Enoksen, E., Shalfawi, S.A., \& Tønnessen, E. (2011). The effect of high- vs. low-intensity training on aerobic capacity in well-trained male middle-distance runners. Journal of Strength and Conditioning Research, 25(3), 812-818.

Garnacho-Castaño, M.V., Domínguez, R., Ruiz-Solano, P., \& Maté-Muñoz, J.L. (2015). Acute physiological and mechanical responses during resistance exercise at the lactate threshold intensity. Journal of Strength and Conditioning Research, 29(10), 2867-2873.

Girard, O., Millet, G., Micallef, J.-P., \& Racinais, S. (2012). Alteration in neuromuscular function after a $5 \mathrm{~km}$ running time trial. European Journal of Applied Physiology, 112(6), 2323-2330.

Guglielmo, L., Greco, C., \& Denadai, B. (2009). Effects of strength training on running economy. International Journal of Sports Medicine, 30(1), 27.

Guglielmo, L.G.A., Babel Junior, R.J., Arins, F.B., \& Dittrich, N. (2012). Índices fisiológicos associados com a performance aeróbia de corredores nas distâncias de $1,5 \mathrm{~km}, 3 \mathrm{~km}$ e $5 \mathrm{~km}$. [Physiological indices associated with aerobic performance in the distances of $1.5 \mathrm{~km}, 3 \mathrm{~km}$ and $5 \mathrm{~km}$. In Portuguese.] Motriz, 18(4), 690-698.

Häkkinen, K., Alen, M., Kraemer, W., Gorostiaga, E., Izquierdo, M., Rusko, H., . ., \& Kaarakainen, E. (2003). Neuromuscular adaptations during concurrent strength and endurance training versus strength training. European Journal of Applied Physiology, 89(1), 42-52.

Hanon, C., Thépaut-Mathieu, C., \& Vandewalle, H. (2005). Determination of muscular fatigue in elite runners. European journal of applied physiology, 94(1-2), 118-125.

Hermens, H.J., Freriks, B., Merletti, R., Stegeman, D., Blok, J., Rau, G., ..., \& Hägg, G. (1999). European recommendations for surface electromyography. Roessingh Research and Development, 8(2), 13-54.

Hudgins, B., Scharfenberg, J., Triplett, N.T., \& McBride, J.M. (2013). Relationship between jumping ability and running performance in events of varying distance. Journal of Strength and Conditioning Research, 27(3), 563-567.

Hug, F., Laplaud, D., Lucia, A., \& Grelot, L. (2006). EMG threshold determination in eight lower limb muscles during cycling exercise: A pilot study. International Journal of Sports Medicine, 27(6), 456-462.

Lum, D., Tan, F., Pang, J., \& Barbosa, T.M. (2016). Effects of intermittent sprint and plyometric training on endurance running performance. Journal of Sport and Health Science.

Markovic, G., \& Mikulic, P. (2010). Neuro-musculoskeletal and performance adaptations to lower-extremity plyometric training. Sports Medicine, 40(10), 859-895.

McHugh, M.P. (2003). Recent advances in the understanding of the repeated bout effect: The protective effect against muscle damage from a single bout of eccentric exercise. Scandinavian Journal of Medicine adn Science in Sports, 13(2), 88-97.

Mikkola, J., Vesterinen, V., Taipale, R., Capostagno, B., Häkkinen, K., \& Nummela, A. (2011). Effect of resistance training regimens on treadmill running and neuromuscular performance in recreational endurance runners. Journal of Sports Sciences, 29(13), 1359-1371.

Nummela, A.T., Paavolainen, L.M., Sharwood, K.A., Lambert, M.I., Noakes, T.D., \& Rusko, H.K. (2006). Neuromuscular factors determining $5 \mathrm{~km}$ running performance and running economy in well-trained athletes. European Journal of Applied Physiology, 97(1), 1-8.

Oliveira, A.S., Caputo, F., Aagaard, P., Corvino, R.B., Gonçalves, M., \& Denadai, B.S. (2013). Isokinetic eccentric resistance training prevents loss in mechanical muscle function after running. European Journal of Applied Physiology, 113(9), 2301-2311.

Paavolainen, L., Häkkinen, K., Hämäläinen, I., Nummela, A., \& Rusko, H. (1999). Explosive-strength training improves $5-\mathrm{km}$ running time by improving running economy and muscle power. Journal of Applied Physiology, 86(5), $1527-1533$.

Paschalis, V., Nikolaidis, M.G., Giakas, G., Jamurtas, A.Z., Owolabi, E.O., \& Koutedakis, Y. (2008). Position sense and reaction angle after eccentric exercise: The repeated bout effect. European Journal of Applied Physiology, 103(1), 9-18.

Rønnestad, B.R., \& Mujika, I. (2014). Optimizing strength training for running and cycling endurance performance: A review. Scandinavian Journal of Medicine and Science in Sports, 24(4), 603-612.

Saunders, P.U., Pyne, D.B., Telford, R.D., \& Hawley, J.A. (2004). Factors affecting running economy in trained distance runners. Sports medicine, 34(7), 465-485.

Schumann, M., Pelttari, P., Doma, K., Karavirta, L., \& Häkkinen, K. (2016). Neuromuscular adaptations to samesession combined endurance and strength training in recreational endurance runners. International Journal Sports Medicine, 37(14), 1136-1143. doi: 10.1055/s-0042-112592

Sedano, S., Marín, P.J., Cuadrado, G., \& Redondo, J.C. (2013). Concurrent training in elite male runners: The influence of strength versus muscular endurance training on performance outcomes. Journal of Strength and Conditioning Research, 27(9), 2433-2443. 
Taipale, R., Mikkola, J., Nummela, A., Vesterinen, V., Capostagno, B., Walker, S., . ., \& Häkkinen, K. (2010). Strength training in endurance runners. International Journal of Sports Medicine, 31(07), 468-476.

Taipale, R., Mikkola, J., Vesterinen, V., Nummela, A., \& Häkkinen, K. (2013). Neuromuscular adaptations during combined strength and endurance training in endurance runners: Maximal versus explosive strength training or a mix of both. European Journal of Applied Physiology, 113, 325-335.

Vorup, J., Tybirk, J., Gunnarsson, T.P., Ravnholt, T., Dalsgaard, S., \& Bangsbo, J. (2016). Effect of speed endurance and strength training on performance, running economy and muscular adaptations in endurance-trained runners. European Journal of Applied Physiology, 116, 1331-1341.

Submitted: December 12, 2017

Accepted: November 8, 2019

Published Online First: April 22, 2020

Correspondence to:

Miller Pereira Guimarães, Ph.D.

Department of Physical Education, University of

Lavras

Zip Code: 37200-000, PO BOX 3037 Lavras, Brazil

Phone Number: $+55(35)$ 3829-5132

Email: millerguimaraes@yahoo.com.br

\section{Acknowledgments}

We would like to thank all the participants who selflessly participated in the study. 